\title{
Long-range interactions of excited He atoms with ground-state noble-gas atoms
}

\author{
J.-Y. Zhang, ${ }^{1, *}$ Y. Qian, ${ }^{2}$ Z.-C. Yan, ${ }^{3,4}$ and U. Schwingenschlögl ${ }^{1, \dagger}$ \\ ${ }^{1}$ PSE Division, King Abdullah University of Science and Technology, Thuwal 23955-6900, Saudi Arabia \\ ${ }^{2}$ Supercomputing Laboratory, King Abdullah University of Science and Technology, Thuwal 23955-6900, Saudi Arabia \\ ${ }^{3}$ Department of Physics, University of New Brunswick, Fredericton, New Brunswick, Canada E3B 5A3 \\ ${ }^{4}$ State Key Laboratory of Magnetic Resonance and Atomic and Molecular Physics, Wuhan Institute of Physics and Mathematics, \\ and Center for Cold Atom Physics, Chinese Academy of Sciences, Wuhan 430071, China
}

(Received 29 July 2013; published 9 October 2013)

\begin{abstract}
The dispersion coefficients $C_{6}, C_{8}$, and $C_{10}$ for long-range interactions of $\operatorname{He}\left(n^{1,3} S\right)$ and $\operatorname{He}\left(n^{1,3} P\right), 2 \leqslant n \leqslant 10$, with the ground-state noble-gas atoms $\mathrm{Ne}, \mathrm{Ar}, \mathrm{Kr}$, and $\mathrm{Xe}$ are calculated by summing over the reduced matrix elements of multipole transition operators. The large- $n$ expansions for the sums over the He oscillator strength divided by the corresponding transition energy are presented for these series. Using the expansions, the $C_{6}$ coefficients for the systems involving $\mathrm{He}\left(13^{1,3} \mathrm{~S}\right)$ and $\mathrm{He}\left(13^{1,3} \mathrm{P}\right)$ are calculated and found to be in good agreement with directly calculated values.
\end{abstract}

DOI: 10.1103/PhysRevA.88.044701

PACS number(s): $34.20 . \mathrm{Cf}, 34.50 . \mathrm{Cx}, 34.50 . \mathrm{Fa}$

Collisions of excited noble-gas atoms with atoms and molecules have attracted considerable attention for a long time. The collision processes include Penning ionization [1-5], associative ionization [2], and the formation of quasimolecules [6-8], which are of great importance in many areas of physics, such as gaseous lasers, low-temperature plasmas, astrophysics, and radiation physics. The excited noble-gas atoms can be metastable atoms, optically allowed excited atoms [9], or Rydberg atoms [10-12] such as $\mathrm{He}\left(14^{1} P, 15^{1} P\right.$, $16^{1} P$ ) [10]. A large amount of experimental and theoretical work has been performed to study the collision processes. For theoretical calculations, reliable model potentials are important, as they directly impact the accuracy of the estimated collision characteristics, such as the ionization cross sections and their collisional energy dependences. The long-range part of a model potential plays a key role in determining the rate coefficients for close collision and Penning ionization $[13,14]$. In this work, the dispersion coefficients $C_{6}, C_{8}$, and $C_{10}$ are computed for interactions of the Rydberg series of He with the ground-state noble-gas atoms $\mathrm{Ne}, \mathrm{Ar}, \mathrm{Kr}$, and $\mathrm{Xe}$. Atomic units are used throughout the Brief Report.

The long-range interaction between two atoms is treated as a perturbation to the two isolated atoms. For the systems He-X $(\mathrm{X}=$ ground-state $\mathrm{Ne}, \mathrm{Ar}, \mathrm{Kr}$, and $\mathrm{Xe}$ ), the energy correction can be written in the form

$$
V(R)=-\frac{C_{6}}{R^{6}}-\frac{C_{8}}{R^{8}}-\frac{C_{10}}{R^{10}}-\cdots,
$$

where $C_{k}$ are the dispersion coefficients and $R$ is the interatomic distance. The $C_{k}$ can be calculated by summing over the reduced matrix elements of $2^{\ell}$-pole transition operators [15]. $C_{6}$ can also be represented in terms of products of oscillator strengths $\bar{f}_{n m}^{\ell}$ for $m \rightarrow n$ transitions. For the system He-X with an $S$-symmetry $\mathrm{He}, C_{6}$ is given by

$$
C_{6}=-\frac{3}{2} \sum_{n m} \frac{\bar{f}_{n X}^{1} \bar{f}_{m m_{0}}^{1}}{\Delta E_{n X} \Delta E_{m m_{0}}\left(\Delta E_{n X}+\Delta E_{m m_{0}}\right)},
$$

\footnotetext{
*Junyi.Zhang@kaust.edu.sa

†Udo.Schwingenschlogl@kaust.edu.sa
}

where $\Delta E_{n X}$ is the energy difference between the $n$th state and the ground state of atom $X$, and $\Delta E_{m m_{0}}$ is the energy difference between the $m$ th intermediate state and the initial $m_{0}$ th state of He.

For a highly excited $S$-symmetry He, the main contribution comes from transitions with very small energy spacings, which are compared with the energy spacings of atom $X,\left|\Delta E_{m m_{0}}\right| \ll$ $\Delta E_{n X}$ [16-18]. Therefore, $C_{6}$ can be approximated by the product of the dipole polarizability $\alpha_{1}^{X}$ of the noble-gas atom and the dipole sum $S_{L L_{m_{0}}}^{1}(-1)$ of He $[17,18]$. We thus have

$$
\begin{aligned}
C_{6} & \simeq-\frac{3 \alpha_{1}^{X}}{2} S_{10}^{1}(-1), \\
S_{L L_{m_{0}}}^{1}(-1) & =\sum_{m} \frac{\bar{f}_{m m_{0}}^{1} \delta_{L_{m} L}}{\left(E_{m}-E_{m_{0}}\right)},
\end{aligned}
$$

where $L_{m}$ represents the orbital angular momentum quantum number of the $m$ th state. Similarly, $C_{6}$ for a $P$-symmetry $\mathrm{He}$ can be approximately represented in the form [16]

$C_{6} \simeq-\frac{3 \alpha_{1}^{X}}{4}\left\{\left(4-3 M^{2}\right) S_{01}^{1}(-1)+\frac{22-3 M^{2}}{10} S_{21}^{1}(-1)\right\}$,

where $M=0, \pm 1$ is the magnetic quantum number of the $m_{0}$ th state of $\mathrm{He}$.

For He, the energy spectra and reduced matrix elements of the multipole transition operators are the same as those used for calculating the dispersion coefficients for the low-lying states [19-24] and the polarizabilities of Rydberg states [25]. The wave functions are expanded in terms of Hylleraas-type basis functions,

$$
r_{1}^{i} r_{2}^{j} r_{12}^{k} e^{-\alpha r_{1}-\beta r_{2}} \mathcal{Y}_{\ell_{1} \ell_{2}}^{L M}\left(\hat{\mathbf{r}}_{1}, \hat{\mathbf{r}}_{2}\right)
$$

where $\mathcal{Y}_{\ell_{1} \ell_{2}}^{L M}\left(\hat{\mathbf{r}}_{1}, \hat{\mathbf{r}}_{2}\right)$ is the coupled spherical harmonic forming a common eigenstate of $L^{2}$ and $L_{z}$. Except for some truncations made to avoid near linear dependence, all terms with $i+j+$ $k \leqslant \Omega$ are included, where $\Omega$ is an integer. The nonlinear parameters $\alpha$ and $\beta$ are variationally optimized by the Newton method.

The transition arrays for $\mathrm{Ne}, \mathrm{Ar}, \mathrm{Kr}$, and $\mathrm{Xe}$ are the same as those used for calculating the dispersion coefficients listed 
in Refs. [26-29]. The transition distributions are constrained to satisfy a variety of sum rules [15,30,31]. Moreover, the data employed for the dipole transitions are those previously published by Kumar and Meath [30,31]. Using these data sets, it is expected that the dispersion coefficients $C_{6}$ are accurate within about $1 \%$. The distributions of Mitroy and Zhang [26] are used for the quadrupole and octupole transitions. In addition, the initial sets of distributions are generated by Hartree-Fock calculations. They then are refined by adjusting the excitation energies to reproduce the accurate polarizabilities and dispersion constants computed by many-body perturbation theory [32,33]. Relativistic effects are taken into account for $\mathrm{Kr}$ and $\mathrm{Xe}$ during the adjustment.

Table I presents the coefficients $C_{6}, C_{8}$, and $C_{10}$ for interactions between $\operatorname{He}\left(2^{1,3} S\right)$ metastable states and the ground states of $\mathrm{Ne}, \mathrm{Ar}, \mathrm{Kr}$, and $\mathrm{Xe}$. Comparisons are made with the calculations performed by Bell et al. [34] and by Proctor and Stwalley [18]. Bell et al. have computed the $C_{6}$ coefficients by means of the Casimir-Polder formula [34,35]. For the noble-gas atoms, they employ semiempirical representations of the dynamic dipole polarizabilities $\alpha_{1}(\omega)$ [36,37]. Proctor and Stwalley have evaluated the coefficients $C_{6}, C_{8}$, and $C_{10}$ in terms of series of products of oscillator strength sums $[17,18]$. According to Table I, our $C_{6}$ coefficients are in good agreement with previously published values $[18,34]$. On the other hand, our values of $C_{8}$ and $C_{10}$ are generally larger than those of Proctor and Stwalley, even when taking into account their typical uncertainty of 5\%. Since the transition distributions used in our calculations are tuned to reproduce the quadrupole and octupole polarizabilities, which are more accurate than those used by these authors (compare Table II in Ref. [15] and Table VIII in Ref. [17]), the present calculations should be more accurate.

The coefficients $C_{6}, C_{8}$, and $C_{10}$ for interactions between $\mathrm{He}\left(2{ }^{1} P, 3{ }^{1} P\right)$ and the ground states of $\mathrm{Ne}, \mathrm{Ar}, \mathrm{Kr}$, and $\mathrm{Xe}$ are listed in Table II. Kohmoto and Watanabe [16] have estimated $C_{6}$ for these systems using Eq. (4). For $\mathrm{He}\left(2{ }^{1} P\right)-\mathrm{Ne}, \mathrm{He}\left(2{ }^{1} P\right)-$ $\mathrm{Ar}$, and $\mathrm{He}\left(2{ }^{1} P\right)-\mathrm{Kr}$, the present $C_{6}$ coefficients are $2 \%-5 \%$ larger than the values calculated by Kohmoto and Watanabe, except for the $\Sigma$ state of $\mathrm{He}\left(2^{1} P\right)-\mathrm{Kr}$ where our $C_{6}$ value is about $15 \%$ larger than their result. For $\operatorname{He}\left(2{ }^{1} P\right)$-Xe, our $C_{6}$ values are $4 \%-5 \%$ smaller. For $\mathrm{He}\left(3{ }^{1} P\right)-\mathrm{Ne}$ and $\mathrm{He}\left(3{ }^{1} P\right)-\mathrm{Ar}$, our values are $1 \%-2 \%$ larger, and for $\mathrm{He}\left(3{ }^{1} P\right)-\mathrm{Kr}$, ours are $0.4 \%-0.7 \%$ larger. Finally, for $\mathrm{He}\left(3{ }^{1} P\right)-\mathrm{Xe}$, the $C_{6}$ coefficients determined by the two approaches are virtually the same. It is noted from the above-detailed comparison that, since the assumption $\left|\Delta E_{m m_{0}}\right| \ll \Delta E_{n X}$ is more appropriate for $\mathrm{He}\left(3^{1} P\right)$ than for $\mathrm{He}\left(2^{1} P\right)$, the two kinds of calculations are in better agreement for $\mathrm{He}\left(3{ }^{1} P\right)$ than for $\operatorname{He}\left(2{ }^{1} P\right)$.

The coefficients $C_{6}, C_{8}$, and $C_{10}$ for $\mathrm{He}\left(2{ }^{3} P\right)$ and $\mathrm{He}\left(3{ }^{3} P\right)$ are listed in Table III, while the coefficients for $\operatorname{He}\left(3^{1,3} S\right.$, $\left.n^{1,3} S, n{ }^{1,3} P\right), 4 \leqslant n \leqslant 10$, are presented in the Supplemental Material [38]. The uncertainties of all our $C_{6}$ values are about $1 \%$, mainly due to uncertain distributions of the dipole transitions of noble-gas atoms. The uncertainties in $C_{8}$ and $C_{10}$ are larger. As expected, the absolute contribution of the He continuum to the coefficients $C_{n}$ becomes larger for a rising energy of the initial state. However, the relative contribution becomes significantly smaller, since the density of bound states is higher for an initial state of higher energy

TABLE I. Dispersion coefficients for interactions of $\operatorname{He}\left(2{ }^{1,3} S\right)$ with the ground states of noble-gas atoms Ne, Ar, Kr, and Xe. The numbers in the square brackets denote powers of ten.

\begin{tabular}{|c|c|c|c|}
\hline System & $C_{6}$ (a.u.) & $C_{8}$ (a.u.) & $C_{10}$ (a.u.) \\
\hline $\mathrm{NeHe}\left(2{ }^{1} S\right){ }^{1} \Sigma$ & $8.109[1]$ & $6.644[3]$ & $7.132[5]$ \\
\hline Ref. [18] & $7.752[1] \pm 4.191[-1]$ & $6.281[3] \pm 4.032[1]$ & $6.66[5] \pm 6.751[3]$ \\
\hline Ref. [34] & $8.0[1]$ & & \\
\hline $\operatorname{ArHe}\left(2{ }^{1} S\right){ }^{1} \Sigma$ & $3.286[2]$ & $2.797[4]$ & $3.059[6]$ \\
\hline Ref. [18] & $3.228[2] \pm 4.089[0]$ & $2.695[4] \pm 4.123[2]$ & $2.891[6] \pm 6.650[4]$ \\
\hline Ref. [34] & $3.25[2]$ & & \\
\hline $\operatorname{KrHe}\left(2{ }^{1} S\right){ }^{1} \Sigma$ & $4.938[2]$ & $4.305[4]$ & $4.782[6]$ \\
\hline Ref. [18] & $4.841[2] \pm 8.207[0]$ & $4.038[4] \pm 8.303[2]$ & $4.308[6] \pm 1.334[5]$ \\
\hline Ref. [34] & $4.88[2]$ & & \\
\hline $\mathrm{XeHe}\left(2{ }^{1} S\right){ }^{1} \Sigma$ & $7.891[2]$ & $7.206[4]$ & $8.244[6]$ \\
\hline Ref. [18] & $7.731[2] \pm 1.889[1]$ & $6.343[4] \pm 1.911[3]$ & $6.687[6] \pm 3.065[5]$ \\
\hline Ref. [34] & $7.75[2]$ & & \\
\hline $\mathrm{NeHe}\left(2{ }^{3} S\right){ }^{3} \Sigma$ & $5.646[1]$ & $3.459[3]$ & $2.832[5]$ \\
\hline Ref. [18] & $5.345[1] \pm 5.031[-1]$ & $3.220[3] \pm 3.866[1]$ & $2.592[5] \pm 4.642[3]$ \\
\hline Ref. [34] & $5.6[1]$ & & \\
\hline $\operatorname{ArHe}\left(2{ }^{3} S\right){ }^{3} \Sigma$ & $2.266[2]$ & $1.465[4]$ & $1.236[6]$ \\
\hline Ref. [18] & $2.209[2] \pm 4.909[0]$ & $1.387[4] \pm 3.999[2]$ & $1.138[6] \pm 4.606[4]$ \\
\hline Ref. [34] & $2.26[2]$ & & \\
\hline $\operatorname{KrHe}\left(2{ }^{3} S\right){ }^{3} \Sigma$ & $3.392[2]$ & $2.269[4]$ & $1.958[6]$ \\
\hline Ref. [18] & $3.304[2] \pm 9.852[0]$ & $2.073[4] \pm 8.061[2]$ & $1.690[6] \pm 9.240[4]$ \\
\hline Ref. [34] & $3.37[2]$ & & \\
\hline $\mathrm{XeHe}\left(2{ }^{3} S\right){ }^{3} \Sigma$ & $5.394[2]$ & $3.841[4]$ & $3.457[6]$ \\
\hline Ref. [18] & $5.260[2] \pm 2.268[1]$ & $3.233[4] \pm 1.855[3]$ & $2.599[6] \pm 2.125[5]$ \\
\hline Ref. [34] & $5.33[2]$ & & \\
\hline
\end{tabular}


TABLE II. Dispersion coefficients for interactions of $\mathrm{He}\left(2{ }^{1} P\right.$, $3{ }^{1} \mathrm{P}$ ) with the ground states of noble-gas atoms $\mathrm{Ne}, \mathrm{Ar}, \mathrm{Kr}$, and $\mathrm{Xe}$. The numbers in the square brackets denote powers of ten.

\begin{tabular}{|c|c|c|c|}
\hline System & $C_{6}$ (a.u.) & $C_{8}$ (a.u.) & $C_{10}$ (a.u.) \\
\hline $\mathrm{NeHe}\left(2{ }^{1} P\right){ }^{1} \Sigma$ & $1.134[2]$ & $1.928[4]$ & $2.503[6]$ \\
\hline Ref. [16] & $1.09[2]$ & & \\
\hline $\mathrm{NeHe}\left(2{ }^{1} P\right){ }^{1} \Pi$ & $6.322[1]$ & $8.670[2]$ & $4.637[4]$ \\
\hline Ref. [16] & $6.1[1]$ & & \\
\hline $\operatorname{ArHe}\left(2{ }^{1} P\right){ }^{1} \Sigma$ & $4.502[2]$ & $7.993[4]$ & $1.061[7]$ \\
\hline Ref. [16] & $4.43[2]$ & & \\
\hline $\operatorname{ArHe}\left(2{ }^{1} P\right){ }^{1} \Pi$ & $2.509[2]$ & $4.723[3]$ & $2.506[5]$ \\
\hline Ref. [16] & $2.43[2]$ & & \\
\hline $\operatorname{KrHe}\left(2{ }^{1} P\right)^{1} \Sigma$ & $7.844[2]$ & $1.215[5]$ & $1.641[7]$ \\
\hline Ref. [16] & $6.85[2]$ & & \\
\hline $\operatorname{KrHe}\left(2{ }^{1} P\right){ }^{1} \Pi$ & $4.023[2]$ & $8.104[3]$ & $4.479[5]$ \\
\hline Ref. [16] & $3.83[2]$ & & \\
\hline $\mathrm{XeHe}\left(2{ }^{1} P\right)^{1} \Sigma$ & $1.070[3]$ & $1.960[5]$ & $2.768[7]$ \\
\hline Ref. [16] & $1.113[3]$ & & \\
\hline $\mathrm{XeHe}\left(2{ }^{1} P\right){ }^{1} \Pi$ & $5.934[2]$ & $1.521[4]$ & $9.321[5]$ \\
\hline Ref. [16] & $6.22[2]$ & & \\
\hline $\mathrm{NeHe}\left(3{ }^{1} P\right)^{1} \Sigma$ & $6.797[2]$ & $5.598[5]$ & $2.792[8]$ \\
\hline Ref. [16] & $6.73[2]$ & & \\
\hline $\mathrm{NeHe}\left(3{ }^{1} P\right){ }^{1} \Pi$ & $3.875[2]$ & $1.875[4]$ & $4.618[6]$ \\
\hline Ref. [16] & $3.82[2]$ & & \\
\hline $\operatorname{ArHe}\left(3{ }^{1} P\right){ }^{1} \Sigma$ & $2.821[3]$ & $2.319[6]$ & $1.163[9]$ \\
\hline Ref. [16] & $2.797[3]$ & & \\
\hline $\operatorname{ArHe}\left(3{ }^{1} P\right){ }^{1} \Pi$ & $1.602[3]$ & $8.324[4]$ & $1.969[7]$ \\
\hline Ref. [16] & $1.587[3]$ & & \\
\hline $\operatorname{KrHe}\left(3{ }^{1} P\right){ }^{1} \Sigma$ & $4.257[3]$ & $3.513[6]$ & $1.769[9]$ \\
\hline Ref. [16] & $4.234[3]$ & & \\
\hline $\mathrm{KrHe}\left(3{ }^{1} P\right){ }^{1} \Pi$ & $2.419[3]$ & $1.314[5]$ & $3.064[7]$ \\
\hline Ref. [16] & $2.403[3]$ & & \\
\hline $\mathrm{XeHe}\left(3{ }^{1} P\right)^{1} \Sigma$ & $6.882[3]$ & $5.689[6]$ & $2.889[9]$ \\
\hline Ref. [16] & $6.880[3]$ & & \\
\hline $\mathrm{XeHe}\left(3{ }^{1} P\right){ }^{1} \Pi$ & $3.901[3]$ & $2.293[5]$ & $5.241[7]$ \\
\hline Ref. [16] & $3.904[3]$ & & \\
\hline
\end{tabular}

TABLE III. Dispersion coefficients for interactions of $\mathrm{He}\left(2{ }^{3} P\right.$ and $3{ }^{3} P$ ) with the ground states of nobel-gas atoms $\mathrm{Ne}, \mathrm{Ar}, \mathrm{Kr}$, and $\mathrm{Xe}$. The numbers in the square brackets denote powers of ten.

\begin{tabular}{llll}
\hline \hline System & $C_{6}$ (a.u.) & $C_{8}$ (a.u.) & $C_{10}$ (a.u.) \\
\hline $\mathrm{NeHe}\left(2{ }^{3} P\right)^{3} \Sigma$ & $9.246[1]$ & $1.407[4]$ & $1.624[6]$ \\
$\mathrm{NeHe}\left(2{ }^{3} P\right)^{3} \Pi$ & $5.190[1]$ & $6.967[2]$ & $3.188[4]$ \\
$\operatorname{ArHe}\left(2{ }^{3} P\right)^{3} \Sigma$ & $3.763[2]$ & $5.793[4]$ & $6.899[6]$ \\
$\operatorname{ArHe}\left(2{ }^{3} P\right)^{3} \Pi$ & $2.074[2]$ & $3.690[3]$ & $1.754[5]$ \\
$\operatorname{KrHe}\left(2{ }^{3} P\right)^{3} \Sigma$ & $5.666[2]$ & $8.800[4]$ & $1.070[7]$ \\
$\operatorname{KrHe}\left(2{ }^{3} P\right){ }^{3} \Pi$ & $3.102[2]$ & $6.348[3]$ & $3.194[5]$ \\
$\mathrm{XeHe}\left(2{ }^{3} P\right)^{3} \Sigma$ & $9.086[2]$ & $1.438[5]$ & $1.818[7]$ \\
$\mathrm{XeHe}\left(2{ }^{3} P\right)^{3} \Pi$ & $4.925[2]$ & $1.260[4]$ & $6.906[5]$ \\
$\mathrm{NeHe}\left(3{ }^{3} P\right)^{3} \Sigma$ & $6.078[2]$ & $4.517[5]$ & $2.057[8]$ \\
$\mathrm{NeHe}\left(3{ }^{3} P\right)^{3} \Pi$ & $3.461[2]$ & $1.529[4]$ & $3.408[6]$ \\
$\operatorname{ArHe}\left(3{ }^{3} P\right)^{3} \Sigma$ & $2.515[3]$ & $1.870[6]$ & $8.568[8]$ \\
$\operatorname{ArHe}\left(3{ }^{3} P\right)^{3} \Pi$ & $1.428[3]$ & $6.830[4]$ & $1.459[7]$ \\
$\operatorname{KrHe}\left(3{ }^{3} P\right)^{3} \Sigma$ & $3.806[3]$ & $2.834[6]$ & $1.304[9]$ \\
$\operatorname{KrHe}\left(3{ }^{3} P\right)^{3} \Pi$ & $2.158[3]$ & $1.082[5]$ & $2.279[7]$ \\
$\mathrm{XeHe}\left(3{ }^{3} P\right)^{3} \Sigma$ & $6.145[3]$ & $4.591[6]$ & $2.132[9]$ \\
$\mathrm{XeHe}\left(3{ }^{3} P\right)^{3} \Pi$ & $3.479[3]$ & $1.901[5]$ & $3.925[7]$ \\
\hline \hline
\end{tabular}

TABLE IV. $S_{10}^{1}(-1)$ for $\operatorname{He}\left(n^{1,3} S\right)(2 \leqslant n \leqslant 10)$.

\begin{tabular}{lcc}
\hline \hline & Singlet & Triplet \\
\hline $\mathrm{He}(2 S)$ & 21.36970 & 15.20715 \\
$\mathrm{He}(3 S)$ & 114.3326 & 91.41838 \\
$\mathrm{He}(4 S)$ & 374.9145 & 317.7976 \\
$\mathrm{He}(5 S)$ & 937.6301 & 822.5745 \\
$\mathrm{He}(6 S)$ & 1976.811 & 1773.808 \\
$\mathrm{He}(7 S)$ & 3706.856 & 3379.610 \\
$\mathrm{He}(8 S)$ & 6382.174 & 5888.127 \\
$\mathrm{He}(9 S)$ & 10297.18 & 9587.488 \\
$\mathrm{He}(10 S)$ & 15786.26 & 14805.83 \\
$\mathrm{He}(13 S)$ & 45642.24 & 43456.08 \\
Extrapolated & 45642.10 & 43456.21 \\
\hline \hline
\end{tabular}

such that they contribute relatively more than the continuum. For $\mathrm{He}\left(2{ }^{3} S\right)$-Ne and $\mathrm{He}\left(5{ }^{1} S\right)-\mathrm{Ne}$, for example, the absolute contributions to $C_{6}$ are 3.70 a.u. and 13.82 a.u., respectively, while the relative contributions are $6.6 \%$ and $0.4 \%$. The calculated sums $S_{L L_{m_{0}}}^{1}(-1)$ for $\operatorname{He}\left(n^{1,3} S\right)$ and $\operatorname{He}\left(n^{1,3} P\right)$, $2 \leqslant n \leqslant 10$, are presented in Tables IV and V, respectively, where the uncertainties are of the order of $10^{-7}$. The $C_{6}$ values estimated by Eq. (4) for $\mathrm{He}\left(n^{1,3} S\right)$ and $\mathrm{He}\left(n^{1,3} P\right), 3 \leqslant n \leqslant 10$, are presented in Tables VII and VIII, respectively, of the Supplemental Material [38]. For $\operatorname{He}\left(3^{1,3} S\right)$ and $\operatorname{He}\left(3^{1,3} P\right)$, the approximate $C_{6}$ values are $1 \%-2 \%$ larger than those calculated directly. For $\mathrm{He}\left(4^{1,3} S\right)$ and $\mathrm{He}\left(4^{1,3} P\right)$, they are only up to $0.5 \%$ larger. Since the assumption $\left|\Delta E_{m m_{0}}\right| \ll \Delta E_{n X}$ is more appropriate for $n \geqslant 5$, the approximate $C_{6}$ values are more accurate.

$S_{L L_{n}}^{1}\left(n^{s} L_{n}\right)$, which is $S_{L L_{m_{0}}}^{1}(-1)$, for states with $n>10$ is estimated by extrapolating the data in Tables IV and V. The employed polynomial expansions are

$$
\begin{aligned}
S_{10}^{1}\left(n^{1} S\right)= & 1.6666643 n^{4}-0.9322431 n^{3}+0.5480992 n^{2} \\
& -0.3483184 n+0.5353862, \\
S_{10}^{1}\left(n^{3} S\right)= & 1.6668290 n^{4}-1.9823311 n^{3}+1.2688204 n^{2} \\
& -0.7780914 n+0.7694470, \\
S_{01}^{1}\left(n^{1} P\right)= & 0.5555488 n^{4}+0.0270814 n^{3}-0.5565529 n^{2} \\
& -0.0148016 n+0.0383942,
\end{aligned}
$$

TABLE V. $S_{01}^{1}(-1)$ and $S_{21}^{1}(-1)$ for $\operatorname{He}\left(n^{1,3} P\right)(2 \leqslant n \leqslant 10)$.

\begin{tabular}{lccccc}
\hline \hline & \multicolumn{2}{c}{ Singlet } & & \multicolumn{2}{c}{ Triplet } \\
\cline { 2 - 3 } \cline { 6 - 7 } & $S_{01}^{1}(-1)$ & $S_{21}^{1}(-1)$ & & $S_{01}^{1}(-1)$ & $S_{21}^{1}(-1)$ \\
\hline $\mathrm{He}(2 P)$ & 6.892639 & 13.93435 & & 5.688321 & 11.57888 \\
$\mathrm{He}(3 P)$ & 40.71569 & 81.58852 & & 36.35748 & 72.89091 \\
$\mathrm{He}(4 P)$ & 135.0280 & 270.1158 & & 124.2924 & 248.7315 \\
$\mathrm{He}(5 P)$ & 336.6538 & 673.3360 & & 315.2755 & 630.5895 \\
$\mathrm{He}(6 P)$ & 705.7546 & 1411.524 & & 668.4010 & 1336.829 \\
$\mathrm{He}(7 P)$ & 1315.825 & 2631.659 & & 1256.093 & 2512.202 \\
$\mathrm{He}(8 P)$ & 2253.694 & 4507.394 & & 2164.108 & 4328.226 \\
$\mathrm{He}(9 P)$ & 3619.523 & 7239.049 & & 3491.536 & 6983.079 \\
$\mathrm{He}(10 P)$ & 5526.804 & 11053.61 & & 5350.800 & 10701.60 \\
$\mathrm{He}(13 P)$ & 15832.33 & 31569.71 & & 15443.83 & 30711.64 \\
Extrapolated & 15832.32 & 31664.72 & & 15443.82 & 30887.00 \\
\hline
\end{tabular}


TABLE VI. Comparison of $C_{6}$ between the directly calculated values and the values predicted by the asymptotic formulas (the second entry) for $\operatorname{He}\left(13^{1,3} S\right)$ and $\operatorname{He}\left(13^{1,3} P\right)$. The numbers in the square brackets denote powers of ten.

\begin{tabular}{lcccc}
\hline \hline & $\mathrm{Ne}$ & $\mathrm{Ar}$ & $\mathrm{Kr}$ & $\mathrm{Xe}$ \\
\hline $\mathrm{He}\left(13{ }^{1} S\right){ }^{1} \Sigma$ & $1.827[5]$ & $7.587[5]$ & $1.150[6]$ & $1.859[6]$ \\
Estimated & $1.827[5]$ & $7.587[5]$ & $1.150[6]$ & $1.859[6]$ \\
$\mathrm{He}\left(13{ }^{3} S\right)^{3} \Sigma$ & $1.740[5]$ & $7.223[5]$ & $1.094[6]$ & $1.770[6]$ \\
Estimated & $1.740[5]$ & $7.223[5]$ & $1.094[6]$ & $1.770[6]$ \\
$\mathrm{He}\left(13{ }^{1} P\right){ }^{1} \Sigma$ & $2.658[5]$ & $1.104[6]$ & $1.672[6]$ & $2.704[6]$ \\
Estimated & $2.662[5]$ & $1.105[6]$ & $1.675[6]$ & $2.709[6]$ \\
$\mathrm{He}\left(13{ }^{1} P\right){ }^{1} \Pi$ & $1.518[5]$ & $6.301[5]$ & $9.547[5]$ & $1.544[6]$ \\
Estimated & $1.521[5]$ & $6.316[5]$ & $9.570[5]$ & $1.548[6]$ \\
$\mathrm{He}\left(13{ }^{3} P\right)^{3} \Sigma$ & $2.589[5]$ & $1.075[6]$ & $1.629[6]$ & $2.634[6]$ \\
Estimated & $2.597[5]$ & $1.078[6]$ & $1.634[6]$ & $2.642[6]$ \\
$\mathrm{He}\left(13{ }^{3} P\right)^{3} \Pi$ & $1.477[5]$ & $6.133[5]$ & $9.289[5]$ & $1.503[6]$ \\
Estimated & $1.484[5]$ & $6.162[5]$ & $9.336[5]$ & $1.510[6]$ \\
\hline \hline
\end{tabular}

$$
\begin{aligned}
S_{21}^{1}\left(n^{1} P\right)= & 1.1112357 n^{4}+0.0497262 n^{3}-1.0591460 n^{2} \\
& -0.3263700 n+0.7087267 \\
S_{01}^{1}\left(n^{3} P\right)= & 0.5555341 n^{4}-0.1510907 n^{3}-0.5480438 n^{2} \\
& +0.1398819 n-0.0453181, \\
S_{21}^{1}\left(n^{3} P\right)= & 1.1105700 n^{4}-0.2897963 n^{3}-1.1990566 n^{2} \\
& +0.5772455 n-0.1748896 .
\end{aligned}
$$

Using the above expressions, we calculate $S_{10}^{1}\left(13^{1,3} S\right)$, $S_{01}^{1}\left(13^{1,3} P\right)$, and $S_{21}^{1}\left(13^{1,3} P\right)$ and give a comparison with the directly calculated values in the end of Tables IV and V. The agreement is excellent for $S_{10}^{1}\left(13^{1,3} S\right)$ and $S_{01}^{1}\left(13^{1,3} P\right)$, while for $S_{21}^{1}\left(13^{1} P\right)$ and $S_{21}^{1}\left(13^{3} P\right)$, there appear differences of $0.3 \%$ and $0.6 \%$, respectively. Comparison is also made between the directly calculated $C_{6}$ coefficients and those estimated with the extrapolated $S_{L L_{n}}^{1}\left(n^{s} L_{n}\right)$ in Table VI. Overall, the agreement in $C_{6}$ is on the same level as that in $S_{L L_{n}}^{1}\left(n^{s} L_{n}\right)$.

In summary, the dispersion coefficients $C_{6}, C_{8}$, and $C_{10}$ have been calculated for long-range interactions of $\operatorname{He}\left(n^{1,3} S\right)$ and $\mathrm{He}\left(n^{1,3} P\right), 2 \leqslant n \leqslant 10$, with the ground-state atoms $\mathrm{Ne}$, $\mathrm{Ar}, \mathrm{Kr}$, and $\mathrm{Xe}$ by summing over the reduced matrix elements of multipole transition operators. The calculated $C_{8}$ and $C_{10}$ coefficients for $\mathrm{He}\left(2^{1,3} S\right)$ and $C_{6}$ coefficients for $\mathrm{He}\left(2^{1} P\right)$ and $\mathrm{He}\left(3^{1} P\right)$ are more accurate than those reported previously. By fitting the sums $S_{L L_{n}}^{1}\left(n^{s} L_{n}\right), 3 \leqslant n \leqslant 10$, we have obtained approximate polynomial expansions to predict the sums for $n>10$. In addition, the reliability of the polynomial expansions and the predicted $C_{6}$ values have been confirmed by direct calculations.

The authors are thankful for the computational resources provided by HPC of KAUST and ACEnet of Canada. Z.-C.Y. is supported by NSERC of Canada, by the Canadian computing facilities of SHARCnet and ACEnet, and in part by the Chinese Academy of Sciences CAS/SAFEA International Partnership Program for Creative Research Teams.
[1] H. Hotop, T. E. Roth, M.-W. Ruf, and A. J. Yencha, Theor. Chem. Acc. 100, 36 (1998).

[2] K. B. Blagoe, E. Dimova, and G. M. Petrov, J. Quant. Spectrosc. Radiat. Transfer 87, 69 (2004).

[3] N. Kishimoto and K. Ohno, Int. Rev. Phys. Chem. 26, 93 (2007).

[4] T. A. Madison and P. E. Siska, J. Chem. Phys. 131, 134309 (2009).

[5] B. Brunetti, P. Candori, D. Cappelletti, S. Falcinelli, F. Pirani, D. Stranges, and F. Vecchiocattivi, Chem. Phys. Lett. 539, 19 (2012).

[6] R. J. Buenker, H.-P. Liebermann, and A. Z. Devdariani, J. Phys. Chem. 111, 1307 (2007).

[7] A. Z. Devdariani, A. K. Belyaev, A. B. Alekseyev, H.-P. Liebermann, and R. J. Buenker, Mol. Phys. 108, 757 (2010).

[8] A. Z. Devdariani, A. K. Belyaev, A. B. Alekseev, H. P. Liebermann, and R. J. Buenker, Russ. J. Phys. Chem. B 5, 952 (2011).

[9] T. Watanabe and M. Stener, J. Chem. Phys. 121, 9948 (2004).

[10] A. Pesnelle, C. Ronge, M. Perdrix, and G. Watel, Phys. Rev. A 34, 5146 (1986).

[11] M. Reicherts, T. Roth, A. Gopalan, M. Ruf, H. Hotop, C. Desfrançois, and I.-I. Fabrikant, Europhys. Lett. 40, 129 (1997).

[12] E. Y. Buslov and B. A. Zon, Phys. Rev. A 85, 042709 (2012).

[13] E. E. Ferguson, Phys. Rev. 128, 210 (1962).

[14] D. R. Bates, K. L. Bell, and A. E. Kingston, Proc. Phys. Soc. 91, 288 (1967).
[15] J.-Y. Zhang and J. Mitroy, Phys. Rev. A 76, 022705 (2007).

[16] M. Kohmoto and T. Watanabe, J. Phys. Soc. Jpn. 42, 246 (1977).

[17] T. R. Proctor and W. C. Stwalley, J. Chem. Phys. 66, 2063 (1977).

[18] T. R. Proctor and W. C. Stwalley, J. Chem. Phys. 68, 5292 (1978).

[19] J.-Y. Zhang, Z.-C. Yan, D. Vrinceanu, and H. R. Sadeghpour, Phys. Rev. A 71, 032712 (2005).

[20] J.-Y. Zhang, Z.-C. Yan, D. Vrinceanu, J. F. Babb, and H. R. Sadeghpour, Phys. Rev. A 73, 022710 (2006).

[21] J.-Y. Zhang, Z.-C. Yan, D. Vrinceanu, J. F. Babb, and H. R. Sadeghpour, Phys. Rev. A 74, 014704 (2006).

[22] J.-Y. Zhang, Z.-C. Yan, D. Vrinceanu, J. F. Babb, and H. R. Sadeghpour, Phys. Rev. A 76, 012723 (2007).

[23] J.-Y. Zhang, L.-Y. Tang, T.-Y. Shi, Z.-C. Yan, and U. Schwingenschlögl, Phys. Rev. A 86, 064701 (2012).

[24] J.-Y. Zhang, J. Mitroy, Z.-C. Yan, J. F. Babb, H. R. Sadeghpour, and U. Schwingenschlögl, J. Chem. Phys. 138, 134317 (2013).

[25] Z.-C. Yan, Phys. Rev. A 62, 052502 (2000).

[26] J. Mitroy and J.-Y. Zhang, Phys. Rev. A 76, 032706 (2007).

[27] J. Mitroy and J.-Y. Zhang, Mol. Phys. 106, 127 (2008).

[28] J.-Y. Zhang, J. Mitroy, H. R. Sadeghpour, and M. W. J. Bromley, Phys. Rev. A 78, 062710 (2008).

[29] L.-Y. Tang, J.-Y. Zhang, Z.-C. Yan, T.-Y. Shi, and J. Mitroy, J. Chem. Phys. 133, 104306 (2010).

[30] A. Kumar and W. J. Meath, Mol. Phys. 54, 823 (1985). 
[31] A. Kumar and W. J. Meath, Can. J. Chem. 63, 1616 (1985).

[32] A. J. Thakkar, H. Hettema, and P. E. S. Wormer, J. Chem. Phys. 97, 3252 (1992).

[33] C. Hattig and B. A. Hess, J. Phys. Chem. 100, 6243 (1996).

[34] K. L. Bell, A. Dalgarno, and A. E. Kingston, J. Phys. B 1, 18 (1968).

[35] H. B. G. Casimir and D. Polder, Phys. Rev. 73, 360 (1948).
[36] R. J. Bell and A. E. Kingston, Proc. Phys. Soc. (London) 88, 901 (1966).

[37] A. Dalgarno, I. H. Morrison, and R. M. Pengelly, Int. J. Quantum Chem. 1, 161 (1967).

[38] See Supplemental Material at http://link.aps.org/supplemental/ 10.1103/PhysRevA.88.044701 for the long-range interactions of excited $\mathrm{He}$ atoms with ground-state noble-gas atoms. 